\title{
UM ESTUDO QUANTITATIVO ENTRE MÉTODOS DE AMOSTRAGEM EM UMA LINHA DE PRODUÇÃO FARMACÊUTICA, PARA IDENTIFICAÇÃO DO MELHOR MÉTODO DE AMOSTRAGEM
}

\author{
A QUANTITATIVE STUDY BETWEEN SAMPLING METHODS IN A \\ PHARMACEUTICAL PRODUCTION LINE, TO IDENTIFY THE BEST SAMPLING \\ METHOD
}

\author{
Tamires Gebrieli Basilio - tamidanie115@gmail.com \\ Faculdade de Tecnologia (Fatec) - Taquaritinga - SP - Brasil \\ Fabrício Domingues Antonio - fabricio.antonio@fatectq.edu.br \\ Faculdade de Tecnologia (Fatec) - Taquaritinga - SP - Brasil
}

DOI: 10.31510/infa.v17i2.1002

Data de publicação: 18/12/2020

\begin{abstract}
RESUMO
Para encontrar a melhor forma de realizar uma amostragem no setor produtivo é uma das maiores dificuldades encontradas pelas empresas. Para o departamento de qualidade, é de extrema importância medir serviços e produtos para garantir que estejam dentro das especificações de seus clientes. Além de ser um método estatístico para diminuir os erros nas inspeções deve ser um método que atenda as normatizações exigidas. Uma boa e adequada seleção de amostragem, pode apresentar de forma clara uma melhoria na auditoria da qualidade do produto e processo, maximizando assim a probabilidade de acerto e ofertando ao mercado produtos com alto nível de qualidade. Dentro de uma metodologia de amostragem, foi utilizado métodos que atendam as normas NBR 5427 - Guia para utilização da norma e a NBR 5426 - Planos de amostragem e procedimentos na inspeção por atributos. O presente trabalho irá avaliar, os diferentes tipos de amostragem dentro do setor produtivo e pontuar as diferenças entre os resultados estatísticos das mesmas.
\end{abstract}

Palavras-chave: Amostragem. Erro amostral. Tamanho da amostra. Métodos estatísticos. Capabilidade.

\begin{abstract}
To find the best way to carry out sampling in the productive sector is one of the greatest difficulties encountered by companies. For the quality department, it is extremely important to measure services and products to ensure that they are within the specifications of its customers. In addition to being a statistical method to reduce errors in inspections, it must be a method that meets the required standards. A good and adequate selection of sampling, can clearly present an improvement in the audit of the quality of the product and process, thus maximizing the probability of success and offering the market products with a high level of quality. Within a sampling methodology, methods that meet the standards NBR 5427 - Guide for the use of the standard and NBR 5426 - Sampling plans and procedures in the inspection by attributes were used. The present work will evaluate, the different types of sampling within the productive sector and point out the differences between their statistical results.
\end{abstract}


Keywords: Sampling. Sample error. Sample size. Statistical methods. Capability.

\section{INTRODUÇÃO}

$\mathrm{Na}$ indústria farmacêutica, na linha de medicamentos, que hoje é regida pela RDC 301, de 21 de agosto de 2019 fica definido como especificação, sendo todo "documento que descreve em detalhes os requisitos com os quais produtos ou materiais usados ou obtidos durante a fabricação devem atender" e os procedimentos são as "descrições das operações a serem realizadas, das precauções a serem tomadas e das medidas a serem aplicadas, direta ou indiretamente relacionadas com a fabricação de um medicamento".

Dessa forma o produto ganha a classificação de aprovado ou reprovado, quando é inspecionado seguindo os ensaios de controle de qualidade definidos em procedimentos.

O que não se pode afirmar é que a avaliação de alguns produtos que constituem um lote, não excluirá a probabilidade de ainda existirem produtos não conformes.

Para tornar esse processo mais confiável é utilizado a técnica de amostragem, onde a obtenção de resultados é extraída a partir de apenas partes coletadas do processo.

BAILY (2000, p.134, apud, ALMEIDA,M.R 2013, p.2) diz que a checagem de todos os itens de um grande lote é tediosa, consome muito tempo e dinheiro e, em razão dos erros humanos, é mais eficaz a checagem de uma amostra selecionada na base da teoria das probabilidades.

Segundo WOODAL e MONTGOMERY (1999, apud ALMEIDA,M.R, 2013, p.2), a variabilidade é sinônimo de desperdício de dinheiro, tempo e esforços, originado de máquinas mal ajustadas, erros do operador e/ou matérias-primas.

Dessa forma existem várias normas que visam estabelecer métodos de ensaios de controle de qualidade, afim de avaliar as características pré definidas pela qualidade no desenvolvimento de um produto ou processo.

\section{FUNDAMENTAÇÃO TEÓRICA}

\subsection{Controle de Qualidade}

Controle de Qualidade é um sistema que tem a função de medir a qualidade de serviços e produtos de acordo com suas especificações técnicas pré definidas. 
Segundo a RDC 301, de 21 de agosto de 2019, sessão III, artigo 13,

O Controle de Qualidade é a parte das BPF referente à coleta de amostras, às especificações e à execução de testes, bem como à organização, à documentação e aos procedimentos de liberação que asseguram que os testes relevantes e necessários sejam executados, e que os materiais não sejam liberados para uso, ou que produtos não sejam liberados para comercialização ou distribuição, até que a sua qualidade tenha sido considerada satisfatória.

Os controles devem ser realizados por meios de procedimentos aprovados, que tem por objetivo avaliar o desempenho do processo e a conformidade de produtos com as especificações pré definidas.

Segundo Moreira (1999, p. 307) que afirma que a qualidade de produtos é definida em termos da "capacidade de um produto para desempenhar suas funções e incluem a durabilidade geral do produto, sua confiabilidade, precisão, facilidade de operação e vários outros atributos valiosos". Observamos então que qualidade abrange tanto os aspectos objetivos, as especificações técnicas do produto, durabilidade e precisão, por exemplo.

\subsection{Amostragem}

Primeiramente define - se população, como o conjunto de indivíduos ou objeto em estudo. Ou seja, são os elementos que estão sob análise e amostra é o subconjunto da população.

O processo de amostragem, consiste na escolha da amostra. Esta por sua vez, estuda as relações existentes entre uma população e as amostras retiradas dessa população. A amostragem é marco inicial e grandemente importante para um estudo estatístico de um processo.

Correa (2003, p. 10), a fase inicial de uma pesquisa, é a coleta de dados. E é sobre essa coleta de dados da amostra que os estudos se desenvolvem com o objetivo de fazer interferências ou modificações sobre a população.

Segundo Guimarães (2008), [...é impraticável observar toda uma população, seja pelo custo alto, seja por dificuldades operacionais.], por esse motivo então examina - se uma amostra que seja representativa ao processo, para que os resultados obtidos possam demostrar toda a população.

Uma vez que conclusões são tiradas por meio de uma amostra, essa apresenta um grau de incerteza. Para refinar o grau de confiança nos resultados, apresentados por meio de uma amostragem é necessário usar técnicas e procedimentos pré definidos, que instruam quanto ao 
planejamento e o processo de amostragem a ser realizado e também obter inferências sobre a população.

\subsection{Inspeção da Qualidade: métodos de amostragem}

A NBR ISO 8402 ( 1994, p,5 apud, ALMEIDA,M.R, 2013, p.5) define a "Inspeção" como sendo uma "atividade tal como medição, exame, ensaio, verificação com calibres ou padrões, de uma ou mais características de uma entidade, e a comparação dos resultados com requisitos especificados, a fim de determinar se a conformidade para cada uma dessas características é obtida”.

Inspeção da Qualidade é uma das etapas mais importantes para a garantia da qualidade de um processo, ou seja, é a certificação de que o produto ou o processo, chegará ao cliente final dentro de todas as especificações.

Seguindo o guia da NBR 5427: Guia para utilização da norma NBR 5426 - Planos de amostragem e procedimentos na inspeção por atributos.

Inspeção por atributos, é a técnica utilizada para classificar a unidade de produto simplesmente como defeituosa ou não, ou seja, o número de defeitos é contado em relação a especificação.

Segundo as definições presentes na NBR5426/1985, "Unidade de produto, pode ser um artigo simples, um par, um conjunto, uma área, um comprimento, uma operação, um volume, um componente de um produto terminado ou o próprio produto terminado".

Para a realização da inspeção de qualidade, deve - se seguir a sequência de operações apresentadas abaixo na Tabela 1:

Tabela 1 - Sequência de operações

\begin{tabular}{|l||}
\hline \multicolumn{1}{|c|}{ OPERAÇÕES } \\
\hline \hline 1. Determinar o tamanho do lote \\
\hline 2. Escolher o nível de inspeção \\
\hline 3. Determinar o código literal do tamanho da amostra \\
\hline 4. Escolher o plano de amostragem \\
\hline 5. Estabelecer a severidade da inspeção \\
\hline 6. Determinar o tamanho da amostra e o número de aceitação \\
\hline 7. Retirada da amostra \\
\hline 8. Inspeção da amostra \\
\hline
\end{tabular}

Fonte: NBR 5427 - Guia para utilização da norma NBR 5426 - Planos de amostragem e procedimentos na inspeção por atributos (1985) 
Após observar a Tabela 1, e seguir o primeiro item, que é determinar o tamanho do lote, identificar na Tabela 2 - Codificação de amostragem, entre qual intervalo o tamanho do lote obtido se enquadra e escolher o nível de inspeção que será utilizado. Feito isso seguir a amostragem e inspeção de qualidade seguindo a Tabela 3 - Plano de amostragem simples.

Tabela 2 - Codificação de amostragem

\begin{tabular}{|c|c|c|c|c|c|c|c|}
\hline Tamanho do lote & $\mathrm{S} 1$ & $\mathrm{~S} 2$ & S3 & S4 & $\mathrm{I}$ & II & III \\
\hline 2 a 8 & $\mathrm{~A}$ & $\mathrm{~A}$ & $\mathrm{~A}$ & A & $\mathrm{A}$ & A & $\mathrm{B}$ \\
\hline 9 a 15 & $\mathrm{~A}$ & $\mathrm{~A}$ & $\mathrm{~A}$ & $\mathrm{~A}$ & $\mathrm{~A}$ & $\mathrm{~B}$ & $\mathrm{C}$ \\
\hline 16 a 25 & $\mathrm{~A}$ & $\mathrm{~A}$ & $\mathrm{~B}$ & $\mathrm{~B}$ & $\mathrm{~B}$ & $\mathrm{C}$ & $\mathrm{D}$ \\
\hline 26 a 50 & A & B & $\mathrm{B}$ & $\mathrm{C}$ & $\mathrm{C}$ & $\mathrm{D}$ & $\mathrm{E}$ \\
\hline 51 a 90 & $\mathrm{~B}$ & $\mathrm{~B}$ & $\mathrm{C}$ & $\mathrm{C}$ & $\mathrm{C}$ & $\mathrm{E}$ & $\mathrm{F}$ \\
\hline 91 a 150 & $\mathrm{~B}$ & $\mathrm{~B}$ & $\mathrm{C}$ & $\mathrm{D}$ & $\mathrm{D}$ & $\mathrm{F}$ & $\mathrm{G}$ \\
\hline 151 a 280 & $\mathrm{~B}$ & $\mathrm{C}$ & $\mathrm{D}$ & $\mathrm{E}$ & $\mathrm{E}$ & $\mathrm{G}$ & $\mathrm{H}$ \\
\hline 281 a 500 & $\mathrm{~B}$ & $\mathrm{C}$ & $\mathrm{D}$ & $\mathrm{E}$ & $\mathrm{F}$ & $\mathrm{H}$ & $\mathrm{J}$ \\
\hline 501 a 1200 & $\mathrm{C}$ & $\mathrm{C}$ & $\mathrm{E}$ & $\mathrm{F}$ & $\mathrm{G}$ & $\mathrm{J}$ & $\mathrm{K}$ \\
\hline 1201 a 3200 & $\mathrm{C}$ & $\mathrm{D}$ & $\mathrm{E}$ & G & $\mathrm{H}$ & $\mathrm{K}$ & $\mathrm{L}$ \\
\hline 3201 a 10000 & $\mathrm{C}$ & $\mathrm{D}$ & $\mathrm{F}$ & $\mathrm{G}$ & $\mathrm{J}$ & $\mathrm{L}$ & $\mathrm{M}$ \\
\hline 10001 a 35000 & $\mathrm{C}$ & $\mathrm{D}$ & $\mathrm{F}$ & $\mathrm{H}$ & $\mathrm{K}$ & $\bar{M}$ & $\mathrm{~N}$ \\
\hline 35001 a 150000 & $\mathrm{D}$ & $\mathrm{E}$ & $\mathrm{G}$ & $\mathrm{J}$ & $\mathrm{L}$ & $\mathrm{N}$ & $\mathrm{P}$ \\
\hline 150001 a 500000 & $\mathrm{D}$ & $\mathrm{E}$ & $\mathrm{G}$ & $\mathrm{J}$ & $\mathrm{M}$ & $\mathrm{P}$ & $\mathrm{Q}$ \\
\hline Acima de 500001 & $\mathrm{D}$ & $\mathrm{E}$ & $\mathrm{H}$ & $\mathrm{K}$ & $\mathrm{N}$ & $\mathrm{Q}$ & $\mathrm{R}$ \\
\hline
\end{tabular}

Fonte: NBR 5426 - Planos de amostragem e procedimentos na inspeção por atributos (1985) 
Tabela 3 - Plano de amostragem simples

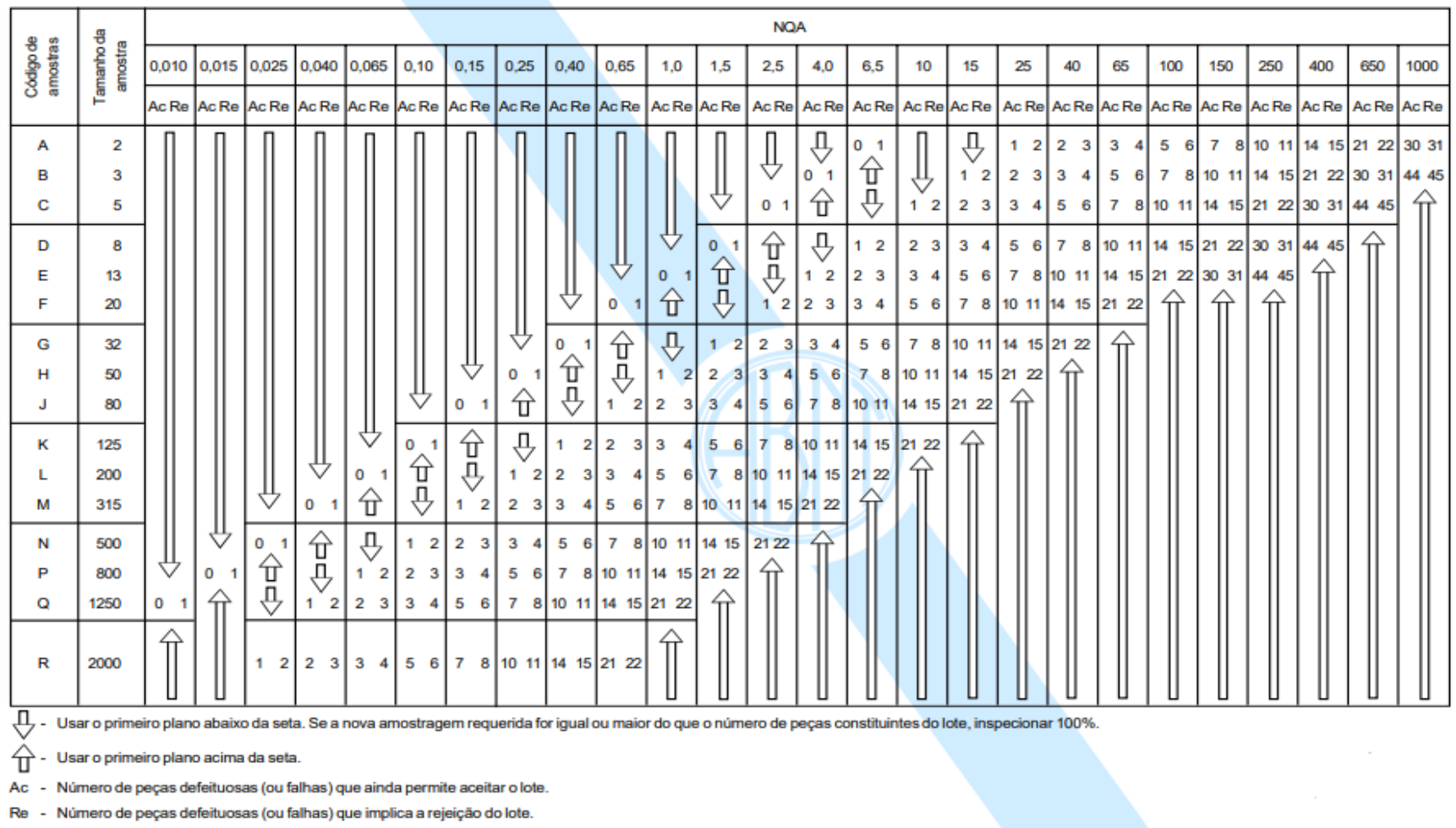

Fonte: NBR 5426 - Planos de amostragem e procedimentos na inspeção por atributos (1985)

Sendo a implantação deste guia na rotina de trabalho de amostragem, o mesmo indica a utilização do nível de inspeção II, amostragem simples com um valor de Nível de Qualidade Aceitável (NQA) de 2,5\%. Para que todas essas indicações sejam utilizadas é preciso analisar o processo em que será utilizada, já que os níveis gerais de inspeção gerais, que são de I a III são utilizados em inspeção do tipo não - destrutiva, ou seja as amostras retiradas podem voltar ao processo, não gerando uma perda produtiva. Já os níveis que vão do S-1 ao S-4, são utilizados para processos que tem fim destrutivo, ou seja, a amostra retirada não voltará ao processo produtivo, acarretando um custo elevado.

\subsection{Controle estatístico do processo (CEP)}

O Controle Estatísticos de Processo (CEP) por sua vez é citado por SLACK, N. (2002), como o monitoramento de um produto ou serviço durante seu processo de produção, pois caso apresente problemas, seu procedimento será interrompido para que as falhas sejam sanadas e o mesmo retorne a sua condição normal.

Todavia qualquer processo possui variações e podemos classificá-las em dois grupos:

Causas de variações comuns: São as variações aleatórias e inevitáveis que acontecem dentro de certos limites, sem uma causa sistemática que possa ser eliminada. 
Causas de variações especiais: Acontece quando o processo apresenta desvios sistemáticos ou variações fora dos seus limites, como consequência de motivos claramente identificáveis dentro do processo e que podem ser eliminadas.

Essa ferramenta com base estatística vem em todo esse contexto, auxiliar ao controle de qualidade, nas etapas de processo a completar um ciclo que se inicia com a amostragem para garantir a estabilidade e a melhoria contínua de um processo.

\subsection{Capabilidade do processo: índices de capabilidade}

Segundo MARTINS, Petrônio G. LAUGENI, Fernando Piero (2006) A capabilidade do processo é a habilidade de se gerar produtos dentro de uma faixa de especificação definida pela empresa ou pelo cliente.

O estudo de capabilidade do processo se dá pela análise da comparação da faixa característica, ou seja, os resultados realmente obtidos em relação a faixa de especificação, dada pelo limite inferior e o limite superior.

Visualizando a imagem 1 abaixo, podemos concluir que, para obter uma boa capabilidade, o processo deve estar dentro dos limites de controle.

Imagem 1 - Capabilidade

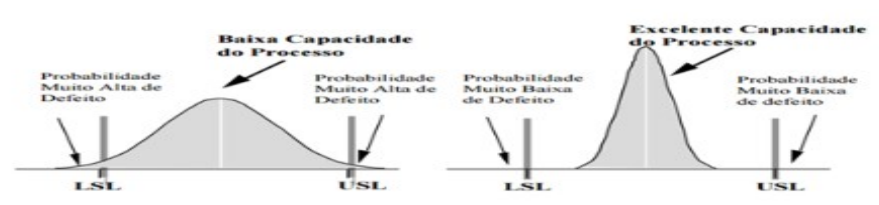

Fonte: MARTINS, P. G.; LAUGENI. F.P,2005.

Conforme Montgomery (1997) e Deleryd (1999), são quatro os índices de capacidade para dados normalmente distribuídos. Estes índices são números adimensionais que permitem uma quantificação do desempenho de processos, sendo eles: Cp, Cpk, Cpm e Cpmk.

O Cp mede a capacidade de maneira simples, para processos centrados. Mede o potencial do processo, já o Cpk mede a capacidade de processos que não estão centrados.

Uma das maneiras, conforme Montgomery (2004) para analisar este índice é definir três intervalos de referência, mostrados na Tabela 4. 
Tabela 4 - Intervalos de referência para análise do índice

\begin{tabular}{lll}
\hline \multicolumn{1}{c}{ CP } & Itens não conformes (PPM) & \multicolumn{1}{c}{ Interpretação } \\
\hline$C_{p}<1$ & Acima de 2700 & Processo incapaz \\
$1 \leq C p \leq 1,33$ & 64 a 2700 & Processo aceitável \\
$C p \geq 1,33$ & Abaixo de 64 & $\begin{array}{l}\text { Processo potencialmente } \\
\text { capaz }\end{array}$ \\
\hline
\end{tabular}

Fonte: Montgomery (2004)

Através dos resultados encontrados é possível interpretar observando se o valor nominal de especificação. Quando $\mathrm{Cp}=\mathrm{Cpk}$ pode - se dizer que o processo está centrado, se $\mathrm{Cp}$ for diferente de $\mathrm{Cpk}$, sabe - se que o processo está descentrado, isto é, que a média não corresponde ao valor nominal das especificações e quanto maior for a diferença entre os resultados, mais descentralizado está o processo.

\section{PROCEDIMENTOS METODOLÓGICOS}

Para realização de uma pesquisa aplicada, foi utilizado normas de amostragem, como por exemplo a ABNT (Associação Brasileira de Normas Técnicas) NBR 5425 de jan/1985, NBR 5426 de jan/1985 e NBR 5427 de jan/1985, das quais possibilitaram a contemplação e compreensão da metodologia de amostragem. Outra característica desse estudo é a questão de a coleta de dados ser exploratória, ou seja, apresentar um estudo de caso, dentro do setor produtivo, de uma indústria farmacêutica. Toda a coleta e organização de dados foi abordada de forma qualitativa, baseadas em estudos matemáticos e estatísticos. Alguns instrumentos tornaram - se relevantes para comprovação da hipótese da pesquisa: um referencial bibliográfico, documental e estudo de caso. A pesquisa bibliográfica se fez necessária para entender todos os trabalhos já apresentados sobre o assunto em questão. O acervo documental é tão importante quanto, pois é através de normas e documentações que traçamos as metodologias a serem utilizadas para o então estudo de caso.

\subsection{Estudo de caso}

A empresa na qual foi desenvolvido o estudo de caso, pertence a um grupo multinacional no ramo de produtos farmacêuticos e desinfecção hospitalar, localizada no interior de São Paulo, e não terá seu nome divulgado. Optou - se por desenvolver esse estudo no setor de envase de medicamentos, pois a demanda produtiva desse setor é grande, podendo 
assim avaliar maiores números de dados. O processo escolhido para a pesquisa exploratória, foi o controle de volume e peso. Nesse processo é realizada pesagens dos recipientes envasados, para garantir que o cliente final receba a quantidade mínima especificada em rotulagem, esse trabalho é realizado pelos apontadores da qualidade.

Atualmente a empresa trabalha com um sistema de amostragem, que não possibilita a representatividade da amostragem, já que o método utilizado não tem bases bibliográficas creditadas. Atualmente para todos os processos de envase, independentemente do tamanho do lote produzido, são retiradas 10 unidades de recipientes a cada 30 minutos.

Como visto anteriormente no referencial teórico e seguindo os passos citados no item 2.3 Inspeção de Qualidade: métodos de amostragem, realizou - se a coleta de dados de um produto, com o tamanho total do lote de 4500 unidades, utilizando os grupos I, II e III, já que o material coletado é de categoria não destrutiva, podendo assim retornar ao processo, evitando perdas.

Durante o período de testes da norma NBR, para amostragem do nível I, foi mantido o mesmo número de recipientes coletados no mesmo intervalo de tempo. Para amostragem do Nível II, foram retirados 20 recipientes, num intervalo de 20 minutos entre as amostragens, já para o Nível III de amostragem, foram retirados 15 recipientes no intervalo de tempo de 10 minutos.

O envase do produto em que os dados foram coletados, teve um tempo de duração do processo de envase de 03 (três) horas e 18 (dezoito) minutos, com um total de 4500 unidades.

Para execução dos gráficos estatísticos de carta de controle e capabilidade, foram utilizadas as três situações de amostragem anteriormente citadas, dentro da mesma norma aplicada demonstradas na Tabela 5. Considerando todas especificações pré definidas para liberação do processo produtivo, que são: Peso mínimo: 1074 g e Peso máximo: 1177 g.

Tabela 5 - Amostragem Nível I,II e III

\begin{tabular}{|c|c|c|c|c|c|}
\hline \multicolumn{6}{|c|}{ OPERAÇÕES } \\
\hline 1 & 2 & 3 & 4 & 5 & 6 \\
\hline \multirow{3}{*}{$\begin{array}{l}4500 \\
\text { un. }\end{array}$} & I & $\mathrm{J}$ & \multirow{3}{*}{ Simples } & \multirow{3}{*}{$\begin{array}{l}\text { NQA } \\
2,5 \%\end{array}$} & $\begin{array}{l}80 \text { unidades amostradas; } \\
5 \text { aceita e } 6 \text { rejeita }\end{array}$ \\
\hline & II & $\mathrm{L}$ & & & $\begin{array}{l}200 \text { unidades amostradas; } \\
10 \text { aceita e } 11 \text { rejeita }\end{array}$ \\
\hline & III & M & & & $\begin{array}{l}315 \text { unidades amostradas; } \\
14 \text { aceita e } 15 \text { rejeita }\end{array}$ \\
\hline
\end{tabular}

Fonte: Os autores (2020) 
Tabela 6 - Carta de Controle e Relatório de Capabilidade - Nível I

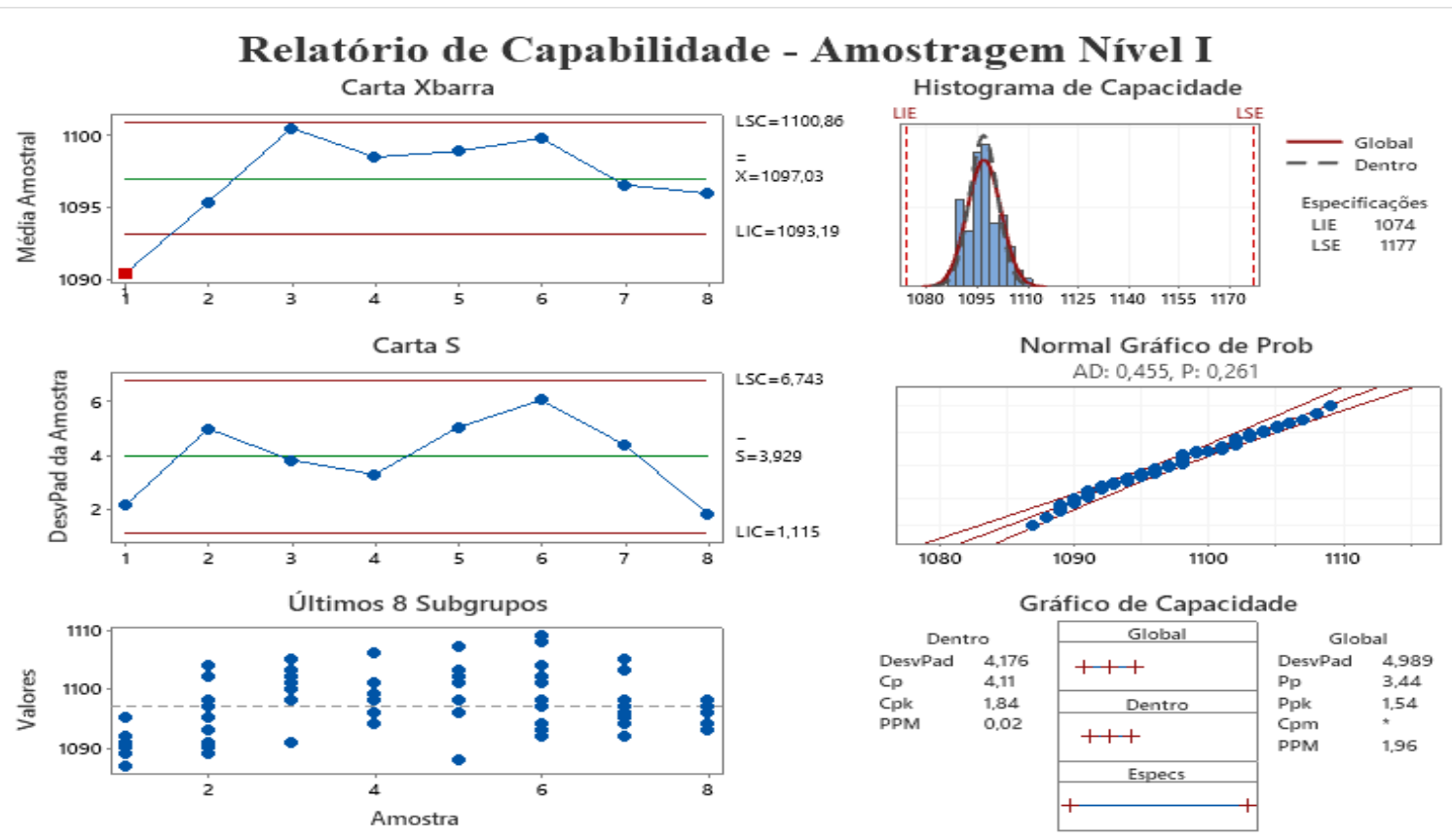

A dispersão do processo real é representada por 6 sigma.

Fonte: Os autores (2020)

Tabela 7 - Carta de Controle e Relatório de Capabilidade - Nível II

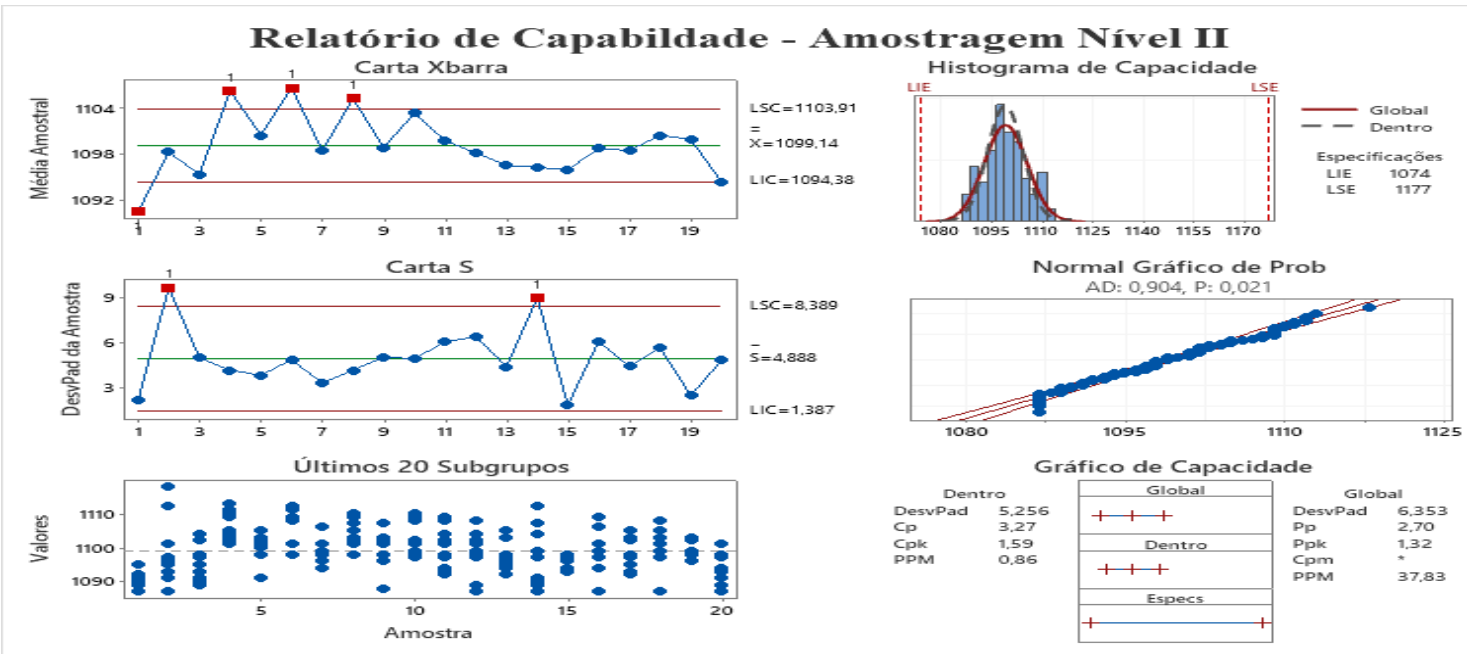

A dispersão do processo real é representada por 6 sigma.

Fonte: Os autores (2020) 
Tabela 8 - Carta Controle e Relatório de Capabilidade - Nível III

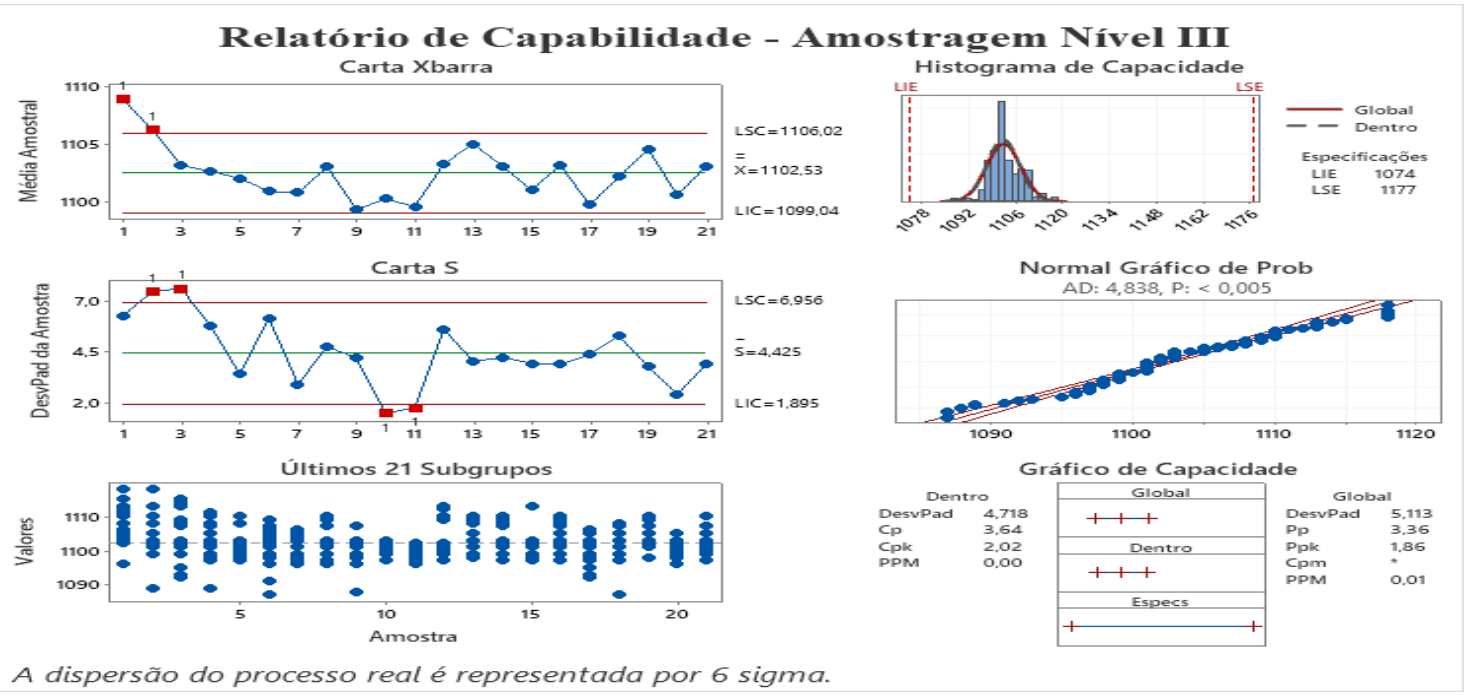

Fonte: Os autores (2020)

\section{RESULTADOS E DISCUSSÃO}

Todos os dados obtidos pelos processos de amostragem foram tratados, através dos gráficos de carta de controle e relatórios de capabilidade dos processos. Obtendo assim os seguintes resultados.

Nível I: Min: 1093g e Máx: $1100 \mathrm{~g}, \mathrm{Cp}: 4,11, \mathrm{Cpk}: 1,84$

Nível II: Min: 1094g e Máx: 1103g, Cp: 3,27, Cpk: 1,59

Nível III: Min: 1099g e Máx: 1106g, Cp: 3,64, Cpk: 2,02

Observando os dados acima, podemos concluir primeiramente, que os limites pré definidos de mínimo e máximo se diferem muito dos limites encontrados através das cartas de controle. Mostrando assim que o processo de envase está oferecendo uma quantidade muito acima do mínimo especificado em rotulagem aos seus clientes, mostrando também um problema de manutenção de máquinas, já que as mesmas estão aferidas para envasar um volume muito superior ao especificado.

Conforme os índices de $\mathrm{Cp}$ obtidos em ambos os níveis de amostragem, podemos afirmar com o auxílio da Tabela 4 - Intervalo de referência para análise do índice de $\mathrm{Cp}$, citada acima, que todos os processos são potencialmente capazes, já que os resultados obtidos ficam acima de 1,33. Também podemos concluir através do índice de Cpk, que em todos os níveis de amostragem apresentados, nenhum dos processos apresentam uma média dos resultados centrados. Pode -se ver que todos tendem ao LIC (limite inferior de especificação), pois os resultados entre Cp e Cpk são diferentes. 
Sendo assim, os três níveis utilizados, trazem um grau de confiança ao processo de amostragem. O que torna o Nível I mais vantajoso é o fato de o número de amostras ser o menor dentre os outros dois, fazendo com que o apontador da qualidade tenha mais tempo hábil para realização das aferições de peso/volume, necessitando assim de menos mão de obra para o processo de amostragem.

\section{CONSIDERAÇÕES FINAIS}

Baseado em uma revisão bibliográfica com aplicação de princípios em um estudo de caso, foram destacados fatores de grande relevância sobre métodos estatísticos para determinar o tamanho das amostras, com o propósito de firmar uma norma com credibilidade frente aos órgãos de inspeção no setor farmacêutico.

Após a identificação do problema, que é realizar uma amostragem representativa baseada em normas reconhecidas, utilizou - se as normas NBR 5426 - Planos de amostragem e procedimentos na inspeção por atributos e NBR 5427 - Guia para utilização da norma NBR 5426 - Planos de amostragem e procedimentos na inspeção por atributos para a coleta dos dados em três níveis de inspeção diferentes.

Hoje a busca por qualidade de produtos e serviços é cada vez mais crescente e a utilização das técnicas de amostragem que garantem a eficiência dos processos é determinante. Por essa razão, o processo de amostragem necessita de planejamento quanto a sua execução.

Todo o conteúdo apresentado neste trabalho, auxilia no processo de amostragem, obtendo assim melhorias nos processos, garantindo a qualidade do produto, não somente a nível organizacional, mais principalmente para o cliente. Consequentemente através do uso de ferramentas da qualidade é possível identificar dentro dos processos possíveis falhas e melhorias.

\section{REFERÊNCIAS}

ALMEIDA, M.R.; CAMPOS, M.C.; NETO, A.L.P.; SILVEIRA, R.R.; Controle Estatístico da Qualidade: Planejamento de Amostragem para Diminuição de Erros de Inspeção. Engenharia de Produção. Salvador; out. 2013. Disponível em: http://www.abepro.org.br/biblioteca/enegep2013 TN STO 178015 22923.pdf. Acesso em: 13 set. 2020. 
CORREA, S.M.B.B. Probabilidade e estatística. $2^{\text {a }}$ ed. Belo Horizonte: PUC Minas Virtual, 2003.

FONSECA, J.S; MARTINS, G.A. Curso de Estatística. 6ª ed. São Paulo: Atlas, 2011.

GUIMARÃES, P. R. B. Métodos Quantitativos Estatísticos. 1ª ed. Curitiba: IESDE Brasil S.A., 2008.

LAUGENI. F. P.; MARTINS, P. G. Administração da Produção. São Paulo: Saraiva, 5 Ed., 2005.

MONTGOMERY, D. C. Introdução ao controle estatístico da qualidade. Rio de Janeiro, $4^{\mathrm{a}}$ ed., 2004.

MOREIRA, J. C. et al. Dicionário de termos de marketing. São Paulo: Atlas, 1999.

NBR 5426, Janeiro de 1985. Planos de amostragem e procedimentos na inspeção por atributos.

NBR 5427, Janeiro de 1985. Guia para utilização da norma NBR 5426 - Planos de amostragem e procedimentos na inspeção por atributos.

NEVES, J.F. et al.Controle Estatístico de Processos. $1^{\text {a }}$ ed. Rio de Janeiro: Dzetta, 2009.

OLIVEIRA, J. B. et al. Análise da Capabilidade de um Processo: Um estudo de caso baseado nos indicadores CP e Cpk. Engenharia de Produção. Belo Horizonte: Enegep, 2011.

PALADINI, E. P. Avaliação Estratégica Da Qualidade. 1 ${ }^{a}$ Edição, São Paulo: Atlas, $2^{\mathrm{a}}$ Tiragem, 2002.

RESOLUÇÃO n³01, de 21 de Agosto de 2019. Dispõe sobre as Diretrizes Gerais de Boas Práticas de Fabricação de Medicamentos.

SLACK, N. Administração de Produção. São Paulo: Atlas, 2002. 